Revista de Matemática: Teoría y Aplicaciones 2001 8(1) : 27-32

CIMPA - UCR - CCSS ISSN: 1409-2433

\title{
DEFORMACIONES ISOMÉTRICAS INFINITESIMALES DE SUPERFICIES MULTIDIMENSIONALES ENSAMBLADAS
}

\author{
Pavel Markov* ${ }^{*}$ Olman Trejos $^{* *}$
}

Recibido: 14 Abril 2000

\begin{abstract}
Resumen
Se introduce el concepto de superficies multidimensionales ensambladas y no se descarta el caso cuando los trozos de las superficies a ensamblar tienen diferentes dimensiones. Se consideran deformaciones infintesimales de estas superficies. En forma muy general, se demuestra que si los trozos de superficies contienen rigidez infinitesimal de primer grado, entonces la superficie ensamblada contiene rigidez infitesimal de primer grado.
\end{abstract}

Palabras clave: Deformaciones infinitesimales, superficies ensambladas, variedad, inmersión, métrica, reper, correper, fibración, subvariedad, difeormorfismo, deformación isométrica, deformación isométrica infinitesimal, campo de deformaciones isométricas infinitesimales, rigidez.

\begin{abstract}
The article introduces the concept of multidimensional pasted surfaces and considers the case of different dimensions in the surfaces to be pasted. Infinitesimal deformations of such surfaces are also considered. In a general way, it is proved that, if the surface pieces have first order infinitesimal rigidity, then the pasted surface contains infinitesimal rigidity of first order.
\end{abstract}

Keywords: Infinitesimal bending, the pasted together surface, manifold, imbedding, the metrics, frame, coframe, bundles, submanifold, diffeomorphism, bending, curving field.

Mathematics Subject Classification: 53C, 57R.

\footnotetext{
${ }^{*}$ Department of Geometry, Faculty of Mathematics, State University of Rostov-na-Donu, Bolshaya Cadoba 105, Rostov-na-Donu, Russia. E-Mail: mpe@donpac.ru

** Department of Geometry, Faculty of Mathematics, State University of Rostov-na-Donu, Bolshaya Cadoba 105, Rostov-na-Donu, Russia \& Escuela de Matemática, Universidad de Costa Rica, 2060 San José, Costa Rica. E-Mail: otrejos@uic.rnd.runnet.ru
} 


\section{Introducción}

A las deformaciones isométricas infinitesimales de las superficies bidimensionales ensambladas del espacio Euclídeo le han dedicado un número suficientemente grande de trabajos (ver [1]- [8]). Interés sobre este tema es detallado en las aplicaciones sobre la teoría de las envolventes (ver [9]). La lista de trabajos sobre la teoría de las deformaciones isométricas infinitesimales de las superficies multidimensionales en los espacios multidimensionales de igual forma es muy amplio (ver [10]- [25]). Desde el punto de vista mecánico la deformación isométrica infinitesimal de una superficie multidimensional se puede interpretar como la deformación de las conexiones de un sistema mecánico cuando la forma de la energía cinética es estacionaria [23]. Sin embargo, a pesar de la gran cantidad de trabajos sobre deformaciones isométricas infinitesimales de las superficies bidimensionales ensambladas y de las superficies multidimensionales suaves, no tenemos conocimiento de algún trabajo que haya considerado las deformaciones isométricas infinitesimales de superficies multidimensionales ensambladas. Además, este problema representa un gran interés, no solo desde el punto de vista matemático, sino también desde el punto de vista de la teoría de los sistemas mecánicos. En adelante definiremos los conceptos generales, relacionados con las superficies multidimensionales ensambladas y con sus deformaciones isométricas infinitesimales, y demostraremos una condición suficiente de la rigidez infinitesimal de estas superficies.

1. Consideremos la variedad $\Gamma, k$-dimensional, $k \geq 1$, Hausdorf orientable, con base numerable, y el espacio $E, \quad m$-dimensional, $m>k$, Euclídeo y afín. La parte vectorial del espacio $E$ la llamaremos de igual forma $E$. Sean $z: \Gamma \rightarrow E$ una $C^{2}$-inmersión e $I(z)$ la métrica en la variedad $\Gamma$, inducida por esta inmersión. En el entorno de cada punto de la variedad $\Gamma$ se define $C^{1}$-coreper $\tau=\left(\tau^{i}\right)_{i=1}^{k}$, en el cual la métrica $I(z)$ tiene forma normal: $I(z)=\sum_{i=1}^{k} \tau^{i} \otimes \tau^{i}$. A través de $\Phi_{j}^{i}$ representaremos las formas de conexión del coreper $\tau$, definidas de forma biunívoca por las siguientes condiciones: $d \tau^{i}=\sum_{j=1}^{k} \tau^{j} \wedge \Phi_{j}^{i}$, $\Phi_{j}^{i}=-\Phi_{i}^{j}$, donde $i, j=1, \ldots, k, \quad d$ es el signo del diferencial exterior. Representemos a través de $\xi=\left(\xi_{i}\right)_{i=1}^{k}$ el reper local, dual al coreper $\tau$, y suponiendo que $e_{i}=\xi_{i}(z)$, para el diferencial de la inmersión $z$ podemos escribir

$$
d z=\sum_{i=1}^{k} \tau^{i} e_{i}, \quad i=1, \ldots, k .
$$

Los campos $e_{i}$ forman un $C^{1}$-reper local de la fibración tangencial de la superficie $z(\Gamma)$ en el espacio $E$.

Sea $\nu=\left(\nu_{\sigma}\right)_{\sigma=1}^{p}, p=m-k$, un reper local ortonormal de la fibración normal de la superficie $z(\Gamma)$. En cada punto $x$ del dominio del coreper $\tau$ el conjunto de vectores $e_{1}(x), \ldots, e_{k}(x), \nu_{1}(x), \ldots, \nu_{p}(x)$ forman una base ortonormal del espacio $E$. La representación de los diferenciales $d e_{i}$ y $d \nu_{\sigma}$ a través de esta base nos conduce a las fórmulas 
de Gauss y Weingarten

$$
d e_{i}=\sum_{j=1}^{k} \Phi_{i}^{j} e_{j}+\sum_{\sigma=1}^{p} \omega_{i}^{\sigma} \nu_{\sigma}, \quad d \nu_{\sigma}=-\sum_{i=1}^{k} \omega_{i}^{\sigma} e_{i}+\sum_{\rho=1}^{p} \kappa_{\sigma}^{\rho} \nu_{\rho}
$$

donde $\omega_{i}^{\sigma}$ son 1-formas continuas, llamadas formas de inmersión y $\kappa_{\sigma}^{\rho}$ son 1-formas continuas, llamadas formas de torsión de la superficie $z(\Gamma), i=1, \ldots, k$, $\sigma, \rho=1, \ldots, p$. Para las formas de inmersión y de torsión las siguientes igualdades son verdaderas $\sum_{i=1}^{k} \omega_{i}^{\sigma} \wedge \tau^{i}=0, \quad \kappa_{\sigma}^{\rho}=-\kappa_{\rho}^{\sigma}$. La primera de estas igualdades implica la simetría de la forma bilineal diferencial $I I^{\sigma}=\sum_{i=1}^{k} \omega_{i}^{\sigma} \otimes \tau^{i}=-d z d \nu_{\sigma}$. Esta forma es la segunda forma principal de la superficie $z(\Gamma)$ con respecto a la normal $\nu_{\sigma}$.

Representemos a través de $S^{2} T^{*}(\Gamma)$ la fibración de las formas simétricas bilineales deferenciales en la subvariedad $\Gamma$, y a $S^{2} T_{x}^{*}(\Gamma)$ su fibra sobre el punto $x \in \Gamma$. La fibra $S^{2} T_{x}^{*}(\Gamma)$ representa el espacio vectorial de dimensión $\frac{k(k+1)}{2}$. Sea $L(x)$ la envolvente lineal en $S^{2} T_{x}^{*}(\Gamma)$, sujetas a las formas $I I^{1}(x), \ldots, I I^{p}(x)$ y $q(x)=\operatorname{dim} L(x)$. El número $q(x)$ representa la dimensión del espacio normal principal de la superficie $z(\Gamma)$ en el punto $x$ [26]. Claro esta, que $q(x) \leq p$. Al punto $x \in \Gamma$ (y de igual forma a su imagen $z(x) \in z(\Gamma)$ ) lo llamaremos punto planado, si $q(x)<p$.

2. Consideremos las $C^{\infty}$-variedades $X^{+}$y $X^{-}$suaves, orientadas, Hausdorff, con bases numerables, de dimensiones $n^{+}$y $n^{-}$y con las fronteras $\partial X^{+}$y $\partial X^{-}$(pueden estar vacías) y las $C^{\infty}$-subvariedades $\Gamma^{+}$y $\Gamma^{-}$de las variedades $X^{+}$y $X^{-}$respectivamente, de dimensiones $k$, donde $1 \leq k<n^{ \pm}$, tales, que existe un $C^{\infty}$-difeomorfismo $\varphi: \Gamma^{+} \rightarrow \Gamma^{-}$(no se descarta el caso cuando $\Gamma^{ \pm} \cap \partial X^{ \pm} \neq \emptyset$ ). El difeomorfismo $\varphi$ lo llamaremos difeomorfismo de ensamblación. Sean $z^{+}: X^{+} \rightarrow E$ y $z^{-}: X^{-} \rightarrow E$ unas $C^{2}$-inmersiones, las cuales satisfacen la siguiente condición:

$$
z^{+}(x)=z^{-}(\varphi(x))
$$

para cada punto $x \in \Gamma^{+}$. Representando a las respectivas superficies $F^{+}=z^{+}\left(X^{+}\right)$y $F^{-}=z^{-}\left(X^{-}\right)$, al conjunto $F=F^{+} \cup F^{-}$llamaremos la superficie, ensamblada por las superficies $F^{+}$y $F^{-}$a lo largo de la superficie $S=z^{+}\left(\Gamma^{+}\right)$. La superficie $S$ la llamaremos superficie de ensamblación.

Consideremos las deformaciones isométricas infinitesimales de las superficies $F^{+}$y $F^{-}$ con los campos de deformaciones $\delta z^{+}$y $\delta z^{-}$respectivamente, que satisfacen la siguiente condición:

$$
\delta z^{-}(\varphi(x))=\delta z^{+}(x)
$$

para cada punto $x \in \Gamma^{+}$. Llamaremos al par $\delta z=\left(\delta z^{+}, \delta z^{-}\right)$campo de deformación isométrica de la superficie ensamblada $F$. Al campo de deformaciones isométricas $\delta z$ lo llamaremos trivial, si para él: $\delta z^{+}=\Omega \cdot z^{+}+\omega$ y $\delta z^{-}=\Omega \cdot z^{-}+\omega$, donde $\Omega$ es un bivector 
arbitrario constante del producto exterior cuadrado $\bigwedge^{2} E$ del espacio $E, \omega$ es un vector arbitrario constante del espacio $E$ y el punto representa el producto interno del bivector por el vector [27]. El campo de deformación de una deformacioń isométrica infinitesimal trivial representa el campo de velocidades, al moverse la superficie en el espacio $E$ como un cuerpo sólido. La superficie ensamblada $F$ se llama infinitesimalmente rígida, si cualquier campo suyo es trivial.

3. El siguiente teorema nos dá un criterio suficiente de rigidez infinitesimal de una superficie ensamblada.

Teorema 1 Si las superficies $F^{+}$y $F^{-}$poseen rigidez infinitesimal de primer grado, y si la superficie de ensamblación $S$ no contiene puntos planados, entonces, la superficie $F=F^{+} \cup F^{-}$es infinitesimalmente rígida.

Demostración: Que las superficies $F^{+}$y $F^{-}$posean rigidez infinitesimal, significa, que sus campos de deformaciones $\delta z^{+}$y $\delta z^{-}$tienen la siguiente forma

$$
\delta z^{+}=\Omega^{+} z^{+}+\omega^{+}, \quad \delta z^{-}=\Omega^{-} z^{-}+\omega^{-},
$$

donde $\Omega^{+}$y $\Omega^{-}$son bivectores constantes, $\omega^{+}$y $\omega^{-}$son vectores constantes. Para demostrar el teorema debemos mostrar, que $\Omega^{+}=\Omega^{-}$y $\omega^{+}=\omega^{-}$.

Representaremos a través de $V^{+}$y $V^{-}$los campos de rotación de las superficies $F^{+}$ y $F^{-}$respectivamente. Ellos se definen biunívocamente con las deformaciones isométricas infinitesimales de estas superficies y las deformaciones de los reperes locales ortonormales, que concuerdan con estas deformaciones isométricas infinitesimales $\left(\nu_{\sigma}^{+}\right)_{\sigma=1}^{p^{+}} \mathrm{y}\left(\nu_{\sigma}^{-}\right)_{\sigma=1}^{p^{-}}$en la fibración normal de estas superficies con las siguientes igualdades

$$
d \delta z^{ \pm}=V^{ \pm} d z^{ \pm}, \quad \delta \nu_{\sigma}^{ \pm}=V^{ \pm} \nu_{\sigma}^{ \pm}
$$

(ver [23]), donde el signo " $\delta$ representa la variación de la magnitud correspondiente. De las condiciones de ensamblación (3) y (4) surge que en la subvariedad $\Gamma^{+}$tienen lugar las siguientes igualdades $d z^{+}(x)=d z^{-}(\varphi(x))$, y $d \delta z^{+}(x)=d \delta z^{-}(\varphi(x))$. De estas igualdades y de la ecuación (6) obtenemos

$$
\left(V^{+}(x)-V^{-}(\varphi(x))\right) d z^{+}(x)=0 .
$$

De la ecuación (5) surge que $V^{+}=\Omega^{+}, \quad V^{-}=\Omega^{-}$. En tal caso, la igualdad anterior se puede escribir de la siguiente forma

$$
\left(\Omega^{+}-\Omega^{-}\right) d z^{+}(x)=0 .
$$

Representemos a través de $\left(\tau^{i}\right)_{i=1}^{k}$ el coreper local en la subvariedad $\Gamma^{+}$, en la cual la métrica de la superficie $z^{+}\left(\Gamma^{+}\right)$tiene forma normal. Utilizando la ecuación (1), podemos escribir esta última igualdad así: $\left(\Omega^{+}-\Omega^{-}\right) \sum_{i=1}^{k} e_{i} \tau^{i}=0$. De la independencia lineal de las formas $\tau^{i}$ surge que

$$
\left(\Omega^{+}-\Omega^{-}\right) e_{i}=0, \quad i=1, \ldots, k .
$$


Derivando esta igualdad y utilizando las fórmulas de Gauss (2) en ella, encontramos que

$$
\left(\Omega^{+}-\Omega^{-}\right) \sum_{\sigma=1}^{p} \omega_{i}^{\sigma} \nu_{\sigma}=0, \quad i=1, \ldots, k, \quad p=m-k .
$$

Multiplicando tensorialmente por $\tau^{i}$ y sumando por el índice $i=1, \ldots, k$, obtenemos

$$
\left(\Omega^{+}-\Omega^{-}\right) \sum_{\sigma=1}^{p} \nu_{\sigma} I I^{\sigma}=0
$$

donde $I I^{\sigma}$ es la segunda forma principal de la superficie de ensamblación $S$ con relación a la normal $\nu_{\sigma}$.

De la ausencia de puntos planados en la superficie de ensamblación $S$ surge la independencia lineal de las formas $I I^{\sigma}$ en la fibra $S^{2} T_{x}^{*}\left(\Gamma^{+}\right)$en cada punto $x \in \Gamma^{+}$. Por tal razón de esta última igualdad tenemos, que

$$
\left(\Omega^{+}-\Omega^{-}\right) \nu_{\sigma}=0, \quad \sigma=1, \ldots, p .
$$

En combinación con la ecuación (7) obtenemos que $\Omega^{+}-\Omega^{-}=0$. De las igualadades (5) y de las condiciones de ensamblación (3) y (4) surge que $\omega^{+}=\omega^{-}$. El teorema queda demostrado.

\section{Referencias}

[1] Vekua, I.N. (1998) Funciones Analiticas Generalizadas. M. 510 pp. (en ruso).

[2] Boyarskyi, B.V.; Vekua, I.N. (1958) "Demostración de la rigidez infinitesimal de superficies regionalmente regulares cerradas convexas de curvatura no negativa", Izviestiya AN SSSR. Cien. Mat. 22(2): 165-176 (en ruso).

[3] Boyarskyi, B.V. (1959) "Sobre la rigidez infinitesimal de algunas superficies de ensamblación", Uspieji Matem. Nauk. 14(3): 141-146 (en ruso).

[4] Sabitov, I.J. (1963) "Sobre la rigidez infinitesimal de algunas superficies de revolución", Matem. Sbornik. 60(4): 506-519 (en ruso).

[5] Sabitov, I.J. (1967) "Sobre una condicion de rigidez infinitesimal de las superficies de ensamblación", Matem. Zamietki. 2(1): 105-113 (en ruso).

[6] Fomienko, V.T.; Markov P.E. (1976) "Sobre la rigidez infinitesimal de las supericies reflejadas vipuchennij", Matem. Zamietki. 19(3): 469-479 (en ruso).

[7] Fomienko, V.T.; Markov P.E. (1977) "The rigidity of a class of intrinsically pasted together surfaces", Ukrain. Geometr. Sb. Vyp. 20: 124-132 (en ruso).

[8] Markov, P.E. (1977) "The rigidity of star-shaped inner-sewn surfaces", Math. Notes 22(3-4): 669-675 (en ruso).

[9] Pogorielov, A.V. (1967) Métodos Geométricos en la Teoría No Lineal de las Envolventes Uprugij. M. 280 pp. (en ruso). 
[10] Yanenko, N.N. (1954) "Hacia la teoría de inmersión de superficies en el espacio euclídeo multidimensional", Tr. Mosk. Matem. Obchestva. 3: 89-180 (en ruso).

[11] Moor, J.D. (1971) "Isometric immersions of Riemannian products", J. Differential Geometry 5(1): 159-168.

[12] Tanaka, N. (1972) "Rigidity for elliptic isometric embeddings", Proc. Japan Acad. 48: 370-372.

[13] Jacobowitz, H. (1972) "Implicit theorems and isometric embeddings", Annals of Math. 95(2): $191-225$.

[14] Goldstein, R.; Ryan, P. (1974) "Rigidity and energy", Global Analysis and its Applications 2: $233-243$.

[15] Goldstein, R.; Ryan, P. (1975) "Infinitesimal rigidity theorem for of submanifolds", J. Differential Geometry 10(1,2): 46-60.

[16] Tenenblat, K. (1979) "A rigidity theorem for ther-dimensional submanifolds in euclidean sixspace", J. Differential Geometry 14(2): 187-203.

[17] Tenenblat, K. (1979) "On infinitesimal isometric deformations", Proc. Amer. Math. Soc. 75(2): 269-275.

[18] Nannicini, A. (1980) "Rigiditá infinitesima per le ipersurficie compacte fortemente convesse di $\mathbb{R}^{n+1} "$, Bol. Unione Mat. Ital. 2, suppl.: 181-194.

[19] Markov, P.E. (1980) "Deformaciones isométricas infinitesimales de algunas superficies multidimensionales", Matem. Zamietki. 27(3): 469-479 (en ruso).

[20] Markov, P.E. (1982) "Deformaciones isométricas infinitesimales de orden superior de superficies multidimensionales", Ucr. Geom. Sbornik. 25: 87-94 (en ruso).

[21] Markov, P.E. (1983) "Deformaciones isométricas infinitesimales de una clase de superficies multidimensionales con fronteras", Matem. Sbornik. 121(1): 48-59 (en ruso).

[22] Markov, P.E. (1985) "Sobre una clase de deformaciones isométricas infinitesimales de superficies multidimensionales", Izv. SKNC VSH 4: 22-25 (en ruso).

[23] Markov, P.E. (1987) "Deformaciones isometricas infinitesimales de orden superior de superficies multidimensionales en los espacios de curvatura constantes", Matem. Sbornik. 133(1): 64-85 (en ruso).

[24] Markov, P.E. (1997) "Deformaciones generales analíticas infinitesimales de las inmersiones 1", Izv. Vuzov. Matematica 9: 21-34 (en ruso).

[25] Markov, P.E. (1997) "Deformaciones generales analíticas infinitesimales de las inmersiones 2", Izv. Vuzov. Matematica 11: 41-51 (en ruso).

[26] Pieriepielkin, D.I. (1935) "Curvatura y espacios normales de la variedad $V_{m}$ en $R_{n}$ ", Matem. Sbornik. 42(1-3): 100-120 (en ruso).

[27] Cartan, E. (1936) Geometría de las Espacios de Riemann. M. 244 pp. 